\title{
Macronutrients, dietary fibre and salt intake and compliance with recommendations in women of child-bearing age (WCBA) (18-50y) in Ireland
}

\author{
A. O’Mahony ${ }^{1,2}$, L. Kehoe ${ }^{1}$, E. O’Sullivan ${ }^{2}$, A.P. Nugent ${ }^{3,4}$, B.A. McNulty ${ }^{3}$, A. Flynn ${ }^{1}$ and \\ J. Walton ${ }^{2}$ \\ ${ }^{1}$ School of Food and Nutritional Sciences, University College Cork, Cork, Ireland, \\ ${ }^{2}$ Department of Biological Sciences, Munster Technological University, Cork, Ireland, \\ ${ }^{3}$ UCD Institute of Food and Health, University College Dublin, Belfield, Dublin, Ireland and \\ ${ }^{4}$ Institute for Global Food Security, School of Biological Sciences, Queens University Belfast, UK
}

It is well established that preconceptional health (including nutritional status) is critically important with regard to favourable maternal and infant outcomes in both the short- and long-term. It has been estimated that up to $50 \%$ of pregnancies are unplanned thus optimal nutritional status is important for all women of child-bearing age (WCBA) not just those with pregnancy intentions ${ }^{(1)}$. Interestingly, nutrient recommendations for WCBA are not differentiated by pregnancy intention ${ }^{(2)}$. The objective of this study was to investigate the quality of the diet of WCBA (18-50y) in Ireland with respect to macronutrients, dietary fibre and salt.

Analyses were based on the National Adult Nutrition Survey (NANS) (2008-2010) (www.iuna.net). A 4-day semi-weighed food record was used to collect food and beverage intake data from a nationally representative sample of 1500 adults (487 women (18$50 \mathrm{y})$ ). Nutrient intakes were estimated using UK and Irish food composition tables. The mean daily intakes (MDI) of energy and nutrients were calculated using SPSS ${ }^{\mathrm{TM}}$ Version 26 and compliance with recommendations were assessed using national and international dietary reference values as appropriate.

The MDI of energy in WCBA was $1742 \mathrm{kcal}$, with an average intake of $35 \%$ energy (\%TE) from fat, $44 \% \mathrm{TE}$ from carbohydrate and $16 \% \mathrm{TE}$ from protein. The MDI of total fat (35\% TE) in WCBA was within the reference intake range of 20-35\% TE recommended by EFSA; however, $42 \%$ had intakes exceeding this recommendation ${ }^{(2)}$. The MDI of saturated fat was $14 \%$ TE which exceeds the recommendation of $<10 \%$ TE from the UK DoH ${ }^{(3)}$. The MDI of MUFA $(12 \% \mathrm{TE})$ and PUFA $(6 \% \mathrm{TE})$ were in line with the UK recommendations to provide a population average of 12 and $6 \% \mathrm{TE}$, respectively ${ }^{(3)}$. The MDI of carbohydrate (44\%TE) in WCBA was approaching the reference intake range of $45-60 \% \mathrm{TE}$ recommended by EFSA; however, $59 \%$ of WCBA had intakes of carbohydrate below this recommendation ${ }^{(2)}$. The MDI of free sugar $(9 \% \mathrm{TE})$ exceeded the UK SACN recommendation for a maximum average population intake of $<5 \% \mathrm{TE}^{(4)}$. Furthermore, $38 \%$ of WCBA had a free sugar intake greater than the WHO recommendation of $<10 \% \mathrm{TE}$ and $82 \%$ had an intake greater than the conditional recommendation of $<5 \% \mathrm{TE}^{(5)}$. The MDI of dietary fibre $(17 \mathrm{~g})$ was lower than the adequate intake of $25 \mathrm{~g}(2)$. The MDI of salt from food sources only (excluding discretionary salt) was $5.5 \mathrm{~g}$ which is below the maximum population target of $<6 \mathrm{~g}^{(6)}$ from all sources; however, $30-45 \%$ of salt in the Irish diet is from discretionary sources.

Overall, these findings show that while intakes of macronutrients are generally sufficient in WCBA in Ireland, this population group have high intakes of saturated fat, free sugar and salt and low intakes of dietary fibre compared to recommendations. These findings will be useful in developing strategies to improve intakes of these nutrients for WCBA.

\section{Acknowledgements}

The National Adult Nutrition Survey was funded by the Irish Department of Agriculture, Food and the Marine.

\section{References}

1. Sedgh G, et al. (2014) Stud Fam Plann 45(3), 301-314.

2. European Food Safety Authority (2010) EFSA J 8, 1461

3. Department of Health (1991) HMSO: UK.

4. Scientific Advisory Committee on Nutrition (2015) The Stationery Office: UK.

5. World Health Organisation (2015) WHO: Switzerland.

6. Food Safety Authority of Ireland (2016) FSAI: Ireland. 